\title{
Nash Equilibrium Based Fairness
}

\author{
Hisao Kameda, Eitan Altman, Corinne Touati and Arnaud Legrand
}

\begin{abstract}
There are several approaches of sharing resources among users. There is a noncooperative approach wherein each user strives to maximize its own utility. The most common optimality notion is then the Nash equilibrium. Nash equilibria are generally Pareto inefficient. On the other hand, we consider a Nash equilibrium to be fair as it is defined in a context of fair competition without coalitions (such as cartels and syndicates). We show a general framework of systems wherein there exists a Pareto optimal allocation that is Pareto superior to an inefficient Nash equilibrium. We consider this Pareto optimum to be 'Nash equilibrium based fair.' We further define a 'Nash proportionately fair' Pareto optimum. We then provide conditions for the existence of a Pareto-optimal allocation that is, truly or most closely, proportional to a Nash equilibrium. As examples that fit in the above framework, we consider noncooperative flowcontrol problems in communication networks, for which we show the conditions on the existence of Nash-proportionately fair Pareto optimal allocations.
\end{abstract}

Keywords - Nash equilibrium, Nash equilibrium based fairness, Nash proportionate fairness, flow control, noncooperative game, Pareto optimum and inefficiency, power criterion.

\section{INTRODUCTION}

There exist many systems where multiple independent users, or players, may strive to optimize their own utility unilaterally, which can be modeled as noncooperative games. Given users' decisions, the utilities of all users are determined. We call a situation where the decisions of all users are determined an allocation. The allocation where each user attains its own optimum coincidentally is a Nash equilibrium. For example, communication networks like the Internet are joined by a number of independent users or organizations, like Internet service providers, that make decisions independently. It is natural that these independent users seek their own benefits or utilities noncooperatively. Nash equilibria may, however, be Pareto inefficient (or, simply, inefficient), that is, there may exist another allocation of a system where no users have less benefit and at least one has more benefit than in the Nash equilibrium of the system.

On the other hand, there may exist innumerably many Pareto-optimal allocations. Choosing which of them to achieve can be controversial among users. In contrast, each Nash equilibrium is fair among all users in the sense that it is

H. Kameda is with the Department of Computer Science, University of Tsukuba, Tsukuba Science City, Ibaraki 305-8573, Japan. (E-mail: kameda@cs.tsukuba.ac.jp).

E. Altman is with INRIA Sophia Antipolis, B.P. 93, 06902 Sophia Antipolis, Cedex, France (E-mail: Eitan.Altman@sophia.inria.fr).

C. Touati and A. Legrand are with CNRS and INRIA, LIG Laboratory, 51 Av. J. Kuntzmann, 38330 Montbonnot, France (E-mails: \{corinne.touati, arnaud.legrand\}@imag.fr). achieved by the fair competition (with no coalition ${ }^{1}$ ) among users. Our purpose in this paper is to propose a new paradigm for resource sharing, which is on one hand Pareto efficient, and on the other hand has fairness properties that are related to the Nash equilibrium. Among the Pareto optima, only those that are Pareto-superior to a Nash equilibrium could satisfy all users. We consider those Pareto optima Nash equilibrium based fair. In particular, as the allocations that would make all users feel fairness similar to that of the Nash equilibrium, we consider a group of allocations where each user's utility is proportionately larger than that of a Nash equilibrium. We say that such allocations are Nash proportionately fair with respect to the Nash equilibrium. If we identify a Pareto-optimal Nash-proportionately fair allocation, it will satisfy all users more strongly. If there exists no Pareto-optimal Nash-proportionately fair allocation for a Nash equilibrium, we may consider a Pareto optimal allocation that is most Nash-proportionate among the Pareto optimal allocations superior to the Nash equilibrium.

These fairness concepts seem to be of a character different from already proposed ones [1]-[8]. We describe, in particular, the generalized fairness in more details. A spectrum of fairness notions has been defined [7]. Each particular point in this spectrum is identified by a value of some parameter $\alpha$; computing the throughputs that are obtained for a given $\alpha$ is done through some utility maximization problem where the utility is a function of the parameter $\alpha$. Maxmin fairness is obtained for $\alpha \rightarrow \infty$ whereas proportional fairness is achieved for $\alpha \rightarrow 1$. In this paper, we call the generalized fairness with parameter $\alpha$ ' $\alpha$-fairness.'

As an example of the general framework, this article considers flow-control problems for communication networks with multiple ports of entry and of exit, where each user decides its throughput, that is, the rate of its packets to inject into a network so as to optimize its own performance objective unilaterally. As such an objective, we firstly consider the power that is defined as the throughput divided by the expected delay (the expected delay is the expected time for a packet to pass through the network) [9]. This unilaterally optimized allocation is a Nash equilibrium, the existence of which has been proved [10]. It has also been shown that the Nash equilibrium is always strongly Pareto inefficient, and an allocation that is Pareto superior to it has been identified [10]. We show here the existence of a Nashproportionately fair Pareto-optimal solution corresponding to an inefficient Nash equilibrium. Moreover, we present

\footnotetext{
${ }^{1}$ The fact that competition without coalitions can be considered fair is reflected in laws that exist in many countries against cartels and against monopolies.
} 
another flow-control setting with additive costs (instead of the power criterion) as another example of the general framework of Nash-equilibrium-based fairness. We also show the existence of a Nash-equilibrium-based fair Pareto-optimal solution corresponding to an inefficient Nash equilibrium.

We would like to mention another related research direction that concerns decentralized flow control and that has received much attention in the literature. This is the design of a decentralized pricing mechanism such that the individual optimization faced by each user results in a choice of flow that is Pareto optimal. This line of research goes back to Kelly and an extensive list of publications on this line of research can be found in http://www.statslab.cam.ac.uk/ frank/pf/ and in http://www.statslab.cam.ac.uk/frank/int/. Our work is somewhat different in spirit, since we study a given game problem and do not ask how to render the resulting equilibrium efficient by pricing (note that pricing changes the utilities of users).

There may exist multiple Nash equilibria in a system, and choosing one among the Nash equilibria is beyond the scope of this paper. Then, we consider, separately for each inefficient Nash equilibrium, the Nash-equilibrium-based fairness and the Nash proportionate fairness with respect to the inefficient Nash equilibrium.

\section{Organization of this paper}

The rest of this paper is organized as follows. Section II discusses a general framework of Nash-equilibrium-based fairness. Section III discusses flow-control problems as examples of the properties shown in Section II. Subsections III-B and III-C, respectively, present fairness results for the flow control with each user's utility being the power criterion and with additive costs. Section IV concludes this article.

\section{A General Framework of Nash Equilibrium Based FAIRNESS}

In this section, we first show a general framework where a Nash-equilibrium-based fair Pareto-optimal allocation as defined in the Introduction exists.

\section{A. A General Framework}

Consider a system that has $n$ users, numbered $1,2, \ldots, n$. Denote by $\mathcal{N}$ the set of the users $\{1,2, \ldots, n\}$. Let $U_{i}$ denote the utility of user $i, i \in \mathcal{N}$. Denote by $\boldsymbol{U}$ the vector $\left(U_{1}, U_{2}, \ldots, U_{n}\right) . \boldsymbol{U}$ is an element of the space $\mathbb{R}_{+}^{n}$ where $\mathbb{R}_{+}$denotes the set of nonnegative real numbers. That is, we consider the cases where $U_{i} \geq 0$ for all $i \in \mathcal{N}$. We further consider that the realizable value of $U_{i}$ is bounded for all $i \in \mathcal{N}$. Denote by $\mathcal{S}$ the set of realizable $\boldsymbol{U}$. Consider an allocation, $\boldsymbol{U} \in \mathcal{S}$. Define $\check{\mathcal{S}}_{\boldsymbol{U}}$ to be $\left\{\boldsymbol{U}^{\prime} \mid U_{i}^{\prime} \geq U_{i}, i \in \mathcal{N}, \boldsymbol{U}^{\prime} \in\right.$ $\mathcal{S}\}$. We have the following assumption:

Assumption $\Phi 1$. For a given $\boldsymbol{U} \in \mathcal{S}, \check{\mathcal{S}}_{\boldsymbol{U}}$ is closed and bounded.

We naturally see that if $\mathcal{S}$ is closed and bounded, then for every $\boldsymbol{U} \in \mathcal{S}, \check{\mathcal{S}}_{\boldsymbol{U}}$ is closed and bounded.
Theorem 1: If Assumption $\Phi 1$ holds for a Paretoinefficient allocation $\widetilde{\boldsymbol{U}}$, there exists a Pareto-optimal allocation that is Pareto-superior to $\widetilde{\boldsymbol{U}}$.

[Proof] See [11].

Note that, if $\widetilde{\boldsymbol{U}}$ is an inefficient Nash equilibrium, Theorem 1 shows that there exists a Nash-equilibrium-based fair Pareto optimal allocation for $\widetilde{\boldsymbol{U}}$. Figure 1 illustrates a case where Assumption $\Phi 1$ is violated and Theorem 1 does not hold.

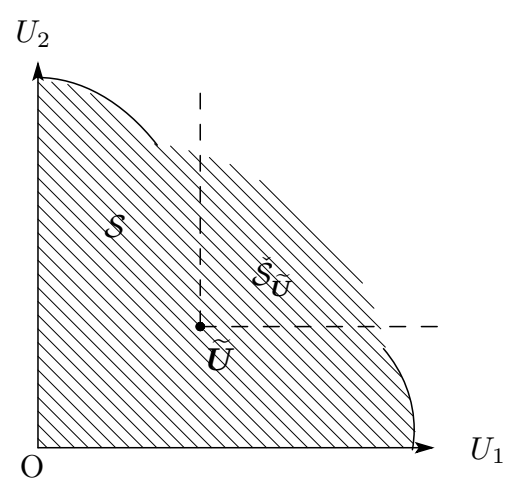

Fig. 1. An example (for $\mathcal{N}=\{1,2\}$ ) of utility sets that violate Assumption $\Phi 1: \check{\mathcal{S}}_{\boldsymbol{U}}$ is not closed, hence there is not always a Pareto-optimal point superior to $\widetilde{\boldsymbol{U}}$.

After we remove all the allocations that are Pareto inefficient from the set $\check{\mathcal{S}}_{\widetilde{U}}$, the set of the remaining allocations is denoted by $\mathcal{S}_{\widetilde{U}}$. Then, from Theorem 1, there remains at least one Pareto-optimal allocation in $\mathcal{S}_{\widetilde{U}}$. We naturally see that $\mathcal{S}_{\widetilde{U}}$ contains all Pareto-optimal allocations that are Pareto superior to the Pareto-inefficient allocation, $\widetilde{\boldsymbol{U}}$. We also note, from the definitions of $\check{\mathcal{S}}_{\widetilde{U}}$ and $\mathcal{S}_{\widetilde{U}}$, that, if an arbitrary Pareto-optimal allocation $\overline{\boldsymbol{U}}$ is Pareto superior to an allocation $\boldsymbol{U} \in \check{\mathcal{S}}_{\widetilde{U}}-\mathcal{S}_{\widetilde{U}}, \bar{U} \in \mathcal{S}_{\widetilde{U}}$ (by noting the definition of $\check{\mathcal{S}}_{\widetilde{U}}$ ) and that, for any allocation $\boldsymbol{U}$ of $\check{\mathcal{S}}_{\widetilde{U}}-\mathcal{S}_{\widetilde{U}}$, there exists a Pareto optimal allocation $\widehat{\boldsymbol{U}} \in \mathcal{S}_{\widetilde{U}}$.

Assumption $\Phi 2$. $\mathcal{S}_{\widetilde{U}}$ is closed and bounded for Pareto inefficient allocation $\widetilde{\boldsymbol{U}}$ in question.

\section{B. Nash Proportionate Fairness}

Among the allocations that are Pareto-superior to an inefficient Nash equilibrium $\widetilde{\boldsymbol{U}}$, we consider a group of allocations where each user's utility is proportionately larger than that of the Nash equilibrium. Note that user $i$ has the utility $\widetilde{U}_{i}$ at the inefficient Nash equilibrium $\widetilde{\boldsymbol{U}}$ for all $i$. Consider an allocation $\overline{\boldsymbol{U}}$. We say that the allocation $\overline{\boldsymbol{U}}$ is Nash proportionate if and only if $\bar{U}_{i}=K \widetilde{U}_{i}$ for some $K \geq 1$ and for all $i$.

If, by increasing the size of $K$, a Pareto-optimal allocation is reached, we may consider that the allocation may satisfy all users in the sense that it reflects the fairness of a Nash equilibrium that is reached by fair competition among users (without unfair coalition) and is Pareto optimal, at the same time. We then call it Nash-proportionately fair Pareto optimum. 
Consider the following measure of Pareto superiority [12]. Denote by $U_{i}^{a}(>0)$ the utility of user $i$ of an allocation $a$ of a system. Assume that the utilities of all users in question have a positive value. Consider that there are two allocations $a$ and $b$ corresponding to two different values of $\boldsymbol{U}$. Denote $\kappa_{i}=U_{i}^{a} / U_{i}^{b}$. If $\min _{i} \kappa_{i}>1$, we can say that $a$ is strongly Pareto superior to $b$. If $\min _{i} \kappa_{i}=1, a$ is Pareto indifferent or Pareto superior to $b$. If $\min _{i} \kappa_{i}<1, a$ is Pareto indifferent or Pareto inferior to $b$. Thus, we use $\kappa=\min _{i} \kappa_{i}$ as a measure of strong Pareto superiority.

Proposition 1: The Nash-proportionately fair Pareto optimal allocation corresponding to a Nash equilibrium, if the latter exists, has the highest Pareto superiority measure with respect to the corresponding Nash equilibrium among all other allocations.

[Proof] See [11].

Consider the case where $\mathcal{S}$ has the boundaries: the hyperplanes $\left\{\boldsymbol{U} \mid U_{i}=0, U_{j} \geq 0(j \neq i)\right\}$ denoted by $\mathcal{B}_{i}$, for all $i \in \mathcal{N}$, and a hypersurface $\eta$ connecting all $\mathcal{B}_{i}, i \in \mathcal{N}$. If the boundary $\eta$ consists of only the Pareto optimal points, clearly, there always exists a Nash-proportionately fair Pareto-optimal point for any inefficient Nash equilibrium. An example of such cases is given in Section III. On the other hand, in the cases where the boundary $\eta$ contains Paretoinefficient points as the system examined by Inoie et al. [13], there may be cases where a Nash-proportionately fair Paretooptimal allocation does not exist for some inefficient Nash equilibria. Note, in passing, Example 1 given later in Section III-B shows the existence of the case where the set of Pareto optimal points that are superior to a Nash equilibrium and that includes the Nash-proportionately fair Pareto-optimal point, when it exists, are separated from the set of generalized $\alpha$-fairness points defined by Mo and Walrand [7].

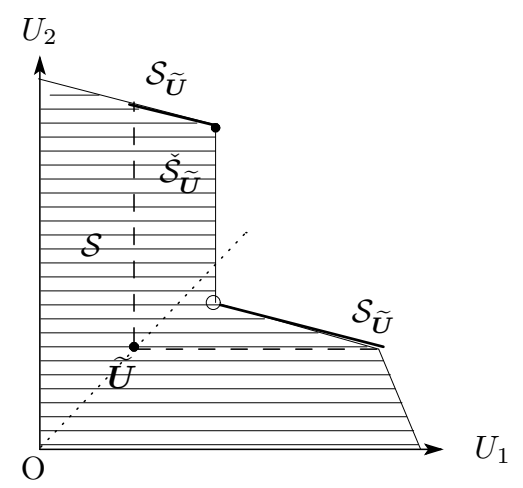

Fig. 2. A typical utility set (for $\mathcal{N}=\{1,2\}$ ) satisfying Assumption $\Phi 1$ but not Assumption $\Phi 2$ : The line $\left\{K \cdot \widetilde{\boldsymbol{U}} \mid K \in \mathbb{R}_{+}\right\}$does not cross the set of Pareto optimal points, and there exists no Pareto-optimal allocation that achieves the Nash proportionate fairness for the Nash equilibrium allocation $\widetilde{\boldsymbol{U}}$ most closely.

\section{The Nash Equilibrium Based Fair Allocation That Is Most Nash Proportionate}

When no Nash proportionately fair Pareto-optimal allocation exists for some inefficient Nash equilibrium, we consider a Pareto-optimal allocation that is Pareto superior to the Nash equilibrium and that achieves Nash proportionate fairness as close as possible. To be more precise, we define an allocation closest to a Nash-proportionately fair Paretooptimal allocation for a Nash equilibrium $\widetilde{\boldsymbol{U}}$ as follows:

If the Nash equilibrium allocation $\widetilde{U}$ is Pareto inefficient, from Theorem 1, we have a set $\mathcal{S}_{\widetilde{U}}$ of Pareto-optimal allocations each element of which is Pareto-superior to it. To each element $\boldsymbol{U}$ of $\mathcal{S}_{\widetilde{U}}$, we assign the real value,

$$
F_{\widetilde{\boldsymbol{U}}}(\boldsymbol{U})=\frac{\sum_{i} \widetilde{U}_{i} U_{i}}{\sqrt{\sum_{i} \widetilde{U}_{i}^{2}} \sqrt{\sum_{i} U_{i}^{2}}},
$$

which gives the value of $\cos \theta$ such that $\theta$ is the angle between the line connecting the origin of the space of $\boldsymbol{U}$ with the Nash equilibrium allocation $\widetilde{\boldsymbol{U}}$ and the line connecting the origin with $\breve{\boldsymbol{U}}$. Naturally, $0 \leq \theta \leq \pi / 2$ and $0 \leq \cos \theta \leq 1$. We note that, as the value of $\cos \theta$ becomes closer to 1 , the value of $\theta$ approaches 0 , and $\breve{U}$ approaches Nash proportionate fairness. If $\theta=0, \check{U}$ achieves Nash proportionate fairness. The allocation $\check{\boldsymbol{U}}$ with the smallest value of $\theta$ achieves Nash proportionate fairness most closely.

Lemma 1: If $\mathcal{S}_{\widetilde{U}}$ is closed and bounded for some Pareto inefficient allocation $\widetilde{\boldsymbol{U}}$, there exists the minimum value of $\theta$ that is associated with a Pareto optimal allocation in $\mathcal{S}_{\widetilde{U}}$.

[Proof] See [11].

We therefore see that the Pareto optimal allocation with the minimum value of $\theta$ in $\mathcal{S}_{\widetilde{U}}$ gives a Nash-proportionately fair allocation to a Pareto inefficient allocation $\widetilde{\boldsymbol{U}}$ most closely. Then, we have the following Proposition.

Proposition 2: If Assumptions $\Phi 1$ and $\Phi 2$ hold, for any inefficient Nash equilibrium allocation, then there exists a Pareto-optimal allocation that achieves the Nash proportionate fairness for the Nash equilibrium allocation most closely.

Figure 2 illustrates a case where Assumption $\Phi 2$ is violated and Proposition 2 does not hold. Figure 3 illustrates a case where both Assumptions $\Phi 1$ and $\Phi 2$ holds and where there exists a Pareto-optimal allocation that achieves the Nash proportionate fairness for the Nash equilibrium allocation most closely, while the Nash proportionately fair Pareto optimum allocation does not exist for the Nash equilibrium allocation in question. In Figures 2 and 3, we note that $\mathcal{S}_{\widetilde{U}}$ is divided into two parts, the upper left and the lower right. In Figure 2, since the boundary connecting the two separate parts of $\mathcal{S}_{\widetilde{U}}$ is a straight line parallel to the vertical axis, then the points consisting the straight line cannot be Pareto optimal points except its top most point. Thus, the leftmost edge of the lower right part of $\mathcal{S}_{\widetilde{U}}$ is open, and then $\mathcal{S}_{\widetilde{U}}$ is not closed, which means the Assumption $\Phi 2$ is not satisfied. In contrast, in Figure 3, both the upper left and lower right parts are closed, and then $\mathcal{S}_{\widetilde{U}}$ is closed, which means that the Assumption $\Phi 2$ is satisfied. We can see the set of achievable utilities in Figure 3 similar to those of the figures 4 and 5 given in [14].

We can easily see that a property similar to the above theorem will hold with any Pareto-inefficient allocation $\breve{U}$ 
for which Assumptions $\Phi 1$ and $\Phi 2$ hold. That is, there exists a Pareto-optimal allocation $\boldsymbol{U}^{\prime}$ that is Pareto superior to the allocation $\breve{U}$ and that equals the value of $\boldsymbol{U}$ that minimizes $F_{\breve{U}}(\boldsymbol{U})$ (defined as (1)).

Remark 1: In this section, we have newly considered the concept of Nash-equilibrium-based fairness. The condition on the existence of a Nash-equilibrium-based fair Pareto optimum has been given. We have shown a general framework in which there exists a Pareto-optimal allocation that achieves the Nash proportionate fairness for an inefficient Nash equilibrium allocation most closely. We note that a number of cases, where the realizable utility set is closed and bounded, have been treated in the literature [2]-[4], [6], [15]-[18]. There have been other cases where the realizable utility set is closed and bounded if the inverse of the cost (or of the mean response time) of each player is regarded as its utility [19]-[21]. Then, Assumption $\Phi 1$ holds, and we may apply Theorem 1 to these cases and show the existence of a Nash-equilibrium-based fair Pareto-optimal allocation for an inefficient Nash equilibrium, if the latter exists. We may apply Proposition 2 to these cases and show the existence of the Pareto optimal allocation that achieves the Nash proportionate fairness most closely, if Assumption $\Phi 2$ holds.

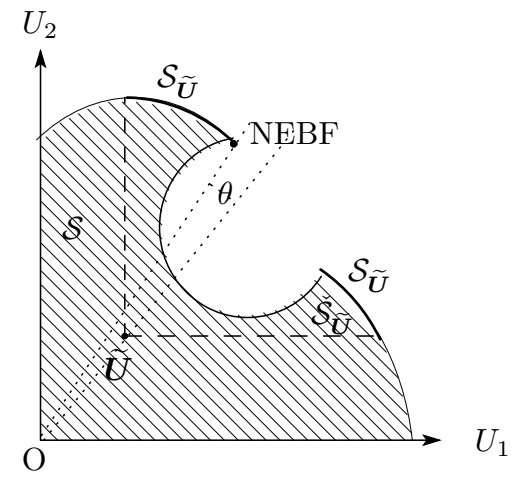

Fig. 3. An example (for $\mathcal{N}=\{1,2\}$ ) of utility sets that satisfy both Assumptions $\Phi 1$ and $\Phi 2$ although the Nash-proportionately fair Pareto optimum allocation does not exist. As Proposition 2 states, there exists a Nash equilibrium based fair (NEBF) Pareto optimum allocation which achieves most closely the Nash-proportionate fairness to the Nash equilibrium $\widetilde{\boldsymbol{U}}$.

\section{Flow Control in Networks}

We examine some flow control problems in networks that fit in both of the above mentioned general frameworks given in Section II. Consider a noncooperative game that has $n$ players each of whom decides the value of $\lambda_{i} \geq 0$, that is, the strategy space consists of nonnegative real numbers. Denote the set of the players $\{1,2, \ldots, n\}$ by $\mathcal{N}$. Thus, the strategy profile is presented by a vector, $\lambda=\left(\lambda_{1}, \lambda_{2}, \ldots, \lambda_{n}\right)$. Let $U_{i}(\lambda)$ denote the utility that player $i$ strives to maximize. (we also allow for values of $-\infty)$. Let $\mathcal{L}$ be the product of the strategy spaces, that is, $\mathcal{L}=\left\{\lambda \mid \lambda_{i} \geq 0, i \in \mathcal{N}\right\}$. Denote by $C(\subset \mathcal{L})$ the set of feasible values of $\lambda$. The definition of feasibility may depend on the system concerned. For example, for a stochastic system, such $\lambda$ for which the system is stable (for example, has a unique stationary regime) is feasible. Such $\lambda$ that leads the system to statistical equilibrium is feasible. $C$ may have boundaries. In the following, we denote by $\widetilde{\lambda}(\in C)$ a strategy profile that presents a Nash equilibrium (with finite utilities).

\section{A. Assumptions on Networks}

Consider a communication network modeled by an open product-form network of $m$ state-independent queues, $k=$ $1,2, \ldots, m$ that model communication links, or, simply, links [22]. Denote the set of the links $\{1,2, \ldots, m\}$ by $\mathcal{M}$. The vertices or nodes connected by links model the routers of the communication network. There are $n$ independent users, $1,2, \ldots, n$ as before. User $i$ decides the feasible rate $\lambda_{i}$ of packets to pass through a communication network so that the utility, $U_{i}$, of the user $i$ may be maximal. $T_{i}$ is the average end-to-end delay of the packets in control of user $i$.

$\mu_{i k}$ is the state-independent service rate of user- $i$ packets at link $k$. In this article, it is assumed that each router (or, node) has a sufficient capacity of storing packets, and, thus, losses of packets may not occur. $q_{i k}$ is the resulting visit rate of user- $i$ packets to link $k$. That is, $q_{i k}$, for all $i, k$, is the solution of the following system of equations:

$$
q_{i k}=p_{0 k}^{i}+\sum_{l} q_{i l} p_{l k}^{i} \text { for all } i \in \mathcal{N}, k \in \mathcal{M},
$$

where $p_{l k}^{i}$ and $p_{0 k}^{i}$, respectively, are the probabilities that a user- $i$ packet goes to link $k$ after leaving link $l$ and when it enters the network, and are fixed and not subject to optimal control. Define $p_{k 0}^{i}=1-\sum_{l} p_{k l}^{i}, \quad i \in \mathcal{N}, k \in \mathcal{M}$. We are concerned only with optimal flow control and not with optimal routing in this paper. Then, if user $i$ injects the rate $\lambda_{i}$ of packets into the network, user- $i$ packets visit link $k$ at the rate of $q_{i k} \lambda_{i}$. User $i$ injects the rate, $p_{0 k}^{i} \lambda_{i}$, of packets into link $k$ from the outside of the network. User- $i$ packets departing from link $k$ leave the network at the frequency (or, probability) $q_{k 0}^{i}$. That is, the network has multiple ports of entry and of exit. Consider the case where the mean response time, $T_{i}^{(k)}$, for a user- $i$ packet to pass through link $k$, is

$$
\begin{gathered}
T_{i}^{(k)}=\mu_{i k}^{-1} T^{(k)} \text { and } T^{(k)}=\frac{1}{1-s_{k} \sum_{p} q_{p k} \lambda_{p} / \mu_{p k}}, \\
\text { if } 1-s_{k} \sum_{p} q_{p k} \lambda_{p} / \mu_{p k}>0, \quad \text { otherwise infinite, }
\end{gathered}
$$

where $s_{k}$ is 1 for a link modeled by a single-server, $1 / h$ for a link consisting of $h$ parallel channels each of which is chosen with probability $1 / h$ and is modeled by a single server, and 0 for a link modeled by an infinite server, for $1-s_{k} \sum_{p} q_{p k} \lambda_{p} / \mu_{p k}>0$ [22]. Denote $\mathcal{K}=\left\{l \mid s_{l} \neq 0\right\}$. Then, using the Little's result,

$$
T_{i}(\lambda)=\sum_{l \in \mathcal{K}} \frac{Q_{i l}}{1-s_{l} \sum_{p} Q_{p l} \lambda_{p}}+\sum_{l \in \mathcal{M}-\mathcal{K}} Q_{i l},
$$

if $1-s_{k} \sum_{p} Q_{p l} \lambda_{p}>0$ for all $l$, otherwise infinite, where $Q_{i l}=\frac{q_{i l}}{\mu_{i l}}$. 
Clearly, $T_{i}(\lambda)$ is increasing in $\lambda$. We note that $\sum_{l \in \mathcal{M}-\mathcal{K}} Q_{i l}$ is constant and independent of the strategy. In order that the statistical equilibrium of this network be attained, it must hold that $\lambda \in C$, where the feasible region $C$ is

$$
C=\left(\lambda \mid \lambda_{i} \geq 0, i \in \mathcal{N}, \text { and } 1-s_{l} \sum_{p} Q_{p l} \lambda_{p}>0, l \in \mathcal{K}\right)
$$

Furthermore, define regions $C^{0}$ and $\bar{C}$ such that

$$
\begin{aligned}
C^{0} & =\left(\lambda \mid \lambda_{i}>0, i \in \mathcal{N}, \text { and } 1-s_{l} \sum_{p} Q_{p l} \lambda_{p}>0, l \in \mathcal{K}\right), \\
\bar{C} & =\left(\lambda \mid \lambda_{i} \geq 0, i \in \mathcal{N}, \text { and } 1-s_{l} \sum_{p} Q_{p l} \lambda_{p} \geq 0, l \in \mathcal{K}\right) .
\end{aligned}
$$

Note that $\bar{C}$ is a closed and bounded subset of $\lambda$. $\partial C$ ( $=\bar{C}-C^{0}$ ) comprises the boundary consisting of $n+k$ hyperplanes each with $(n-1)$-dimensions, $n$ from $\lambda_{i}=0$, $i \in \mathcal{N}$, and $k$ from $1-s_{l} \sum_{p} Q_{p l} \lambda_{p}=0, l \in \mathcal{K}$. We call the part of the boundary consisting of $\lambda_{i}=0$, the $(i-0)$ policy boundary, and the part of boundary which is not any of ( $i-0)$ policy boundary, $i \in \mathcal{K}$, the capacity boundary. Clearly, $\bar{C}$ and $C^{0}$ are convex considering the hyperplanes that define their boundaries.

Each of network users (user- $i$ ) has two important major concerns in choosing the protocol to use: one is the amount of packets user- $i$ can send per unit time (throughput), denoted by $\lambda_{i}$, and the other is the expected time of each packet taken from its origin to its destination (mean response time), denoted by $T_{i}$. As the utility of each user- $i$, we need to consider one scalar value taking account of the above both $\lambda_{i}$ and $T_{i}$. That is, in general we are interested in criteria that will allow us to represent preference to high throughput and to low delay. Both the additive criterion as well as the power criterion fall into this category. More generally, since the Nash equilibrium is unchanged if we replace the utility by the logarithm of the utility, the power criterion can be transformed (using the logarithm) into an additive criterion: the logarithm utility is the sum of the difference between the $\log$ of the throughput and the log of the delay.

In this paper, as such utilities as above, we examine, in particular, the power criterion as in Subsection III-B and the criterion based on some additive costs as in Subsection III-C.

\section{B. Noncooperative Flow Control with the Power Criterion}

The power is defined as $P_{i}=\lambda_{i} / T_{i}$ for a user- $i$ packet. In this subsection, we consider the case where the utility, $U_{i}$, of user $i$ is its power, $P_{i}$, i.e., $U_{i}=P_{i}$ for all $i$. Denote the vector $\left(P_{1}, P_{2}, \ldots, P_{n}\right)$ by $\boldsymbol{P}$. From $(3), P_{i}(\lambda)$ is defined for all $\lambda \in \mathcal{L}$, and $P_{i}(\lambda)=0$ for $\lambda \in \mathcal{L}-C^{0}$ and $i \in \mathcal{N}$. From (3) and the definition $P_{i}=\lambda_{i} / T_{i}$, we see that $\boldsymbol{P}(\lambda)$ is continuous in $\lambda$. By noting that, for $\lambda \in \mathcal{L}-\bar{C}, P_{i}(\lambda)=0$ for all $i$, the set $\mathcal{P}$ of all possible values of $\boldsymbol{P}(\lambda)$ is given by $\lambda \in \bar{C}$. Since $\bar{C}$ is closed and bounded and $\boldsymbol{P}(\lambda)$ is continuous in $\lambda, \mathcal{P}$ is also closed and bounded, that is, Assumption $\Phi 1$ holds for every $\boldsymbol{P} \in \mathcal{P}$. Then, we can apply Theorem 1, and obtain the following.
Corollary 1: For any inefficient Nash equilibrium flow control, there exists a Pareto-optimal flow control that is Pareto superior to the Nash equilibrium flow control. Thus, this Pareto optimum is Nash-equilibrium-based fair.

The existence of a Nash equilibrium flow control, which is inefficient, has been shown [10]. Furthermore, for this example with respect to power optimization, a stronger result, the existence of a Nash-proportionately fair Paretooptimal flow control, will be shown by Theorem 2 .

Assumption $\Phi 3$. Denote a graph $\mathcal{G}$ by $(\mathcal{V}, \mathcal{E})$ such that $\mathcal{V}=$ $\mathcal{N} \cup \mathcal{M}$ and $\mathcal{E}=\left\{(i, k) \mid i \in \mathcal{N}, k \in \mathcal{M}\right.$ and $\left.q_{i k}>0\right\}$. $\mathcal{G}$ is connected.

Theorem 2: If Assumption $\Phi 3$ holds, there exists a Nashproportionately fair Pareto-optimal flow control solution for any inefficient Nash equilibrium of this network.

\section{[Proof] See [11].}

Remark 2: Consider the case where Assumption $\Phi 3$ does not hold. That is, $\mathcal{G}$ is not connected, and consists of multiple disjoint subnetworks, $\mathcal{G}_{1}, \mathcal{G}_{2}, \ldots, \mathcal{G}_{r}$, each of which is connected within itself. Then, Theorem 2 can be applied to each independent subnetwork $\mathcal{G}_{p}, p=1,2, \ldots, r$, and each own Nash-proportionately fair Pareto optimum allocation exists for each disjoint set of users in $\mathcal{G}_{p}, p=1,2, \ldots, r$. It is possible, however, that there does not exist any common Nash-proportionately fair Pareto-optimal flow control allocation for the entire network that violates Assumption $\Phi 3$. We can show such examples by simple models.

Example 1 Consider a simple network consisting of three users $\mathcal{N}=\{1,2,3\}$ and two nodes $\mathcal{K}=\{1,2\}$, where $q_{01}^{1}=$ $q_{02}^{2}=1, q_{01}^{3}=q_{02}^{3}=0.5, q_{10}^{1}=q_{20}^{2}=q_{10}^{3}=q_{20}^{3}=1$, $\mu_{1}^{i}=3(i=1,3)$ for case $\mathrm{A}$ and $\mu_{1}^{i}=30(i=1,3)$ for case $\mathrm{B}$, and $\mu_{2}^{i}=6(i=2,3)$. Each case of the network satisfies Assumption $\Phi 3$.

Recall [7] that, if the utility of user- $i$ is denoted by $U_{i}(\lambda)$ for the strategy profile $\lambda$, the $\alpha$-fairness point $(0 \leq \alpha<\infty)$ is achieved by $\boldsymbol{U}\left(\boldsymbol{\lambda}^{\alpha}\right)=\left(U_{1}\left(\lambda^{\alpha}\right), U_{2}\left(\lambda^{\alpha}\right), \ldots U_{N}\left(\lambda^{\alpha}\right)\right)$ such that

$$
\begin{gathered}
F^{\alpha}\left(\lambda^{\alpha}\right)=\max _{\lambda \in C} F^{\alpha}(\lambda) \\
\text { where } F^{\alpha}(\lambda)=\frac{1}{1-\alpha} \sum_{p}\left\{U_{p}(\lambda)\right\}^{(1-\alpha)} .
\end{gathered}
$$

In Figure 4 , the sizes of utilities $U_{1}(\lambda), U_{2}(\lambda)$, and $U_{3}(\lambda)$ of users 1,2 , and 3 are, respectively, denoted by $U_{1}, U_{2}$, and $U_{3}$. In each of the top and bottom parts of the figure, the curve presents the set of $\alpha$-fairness points for $0 \leq \alpha<\infty$. In each part, the curved surface consisting of the dots, each of which is the outside edge of the very short dotted line, presents the set of Pareto optimal points. There are two X's in each of the top and bottom parts. The left and right X's of each part present, respectively, the Nash equilibrium point (NE) and the Nash-proportionately-fair Pareto optimal point (NPF). In each part, the one dashed line presents a straight line connecting the origin, the NE, and the NPF. We see that, in the network of case A (top), the Nash proportionate 

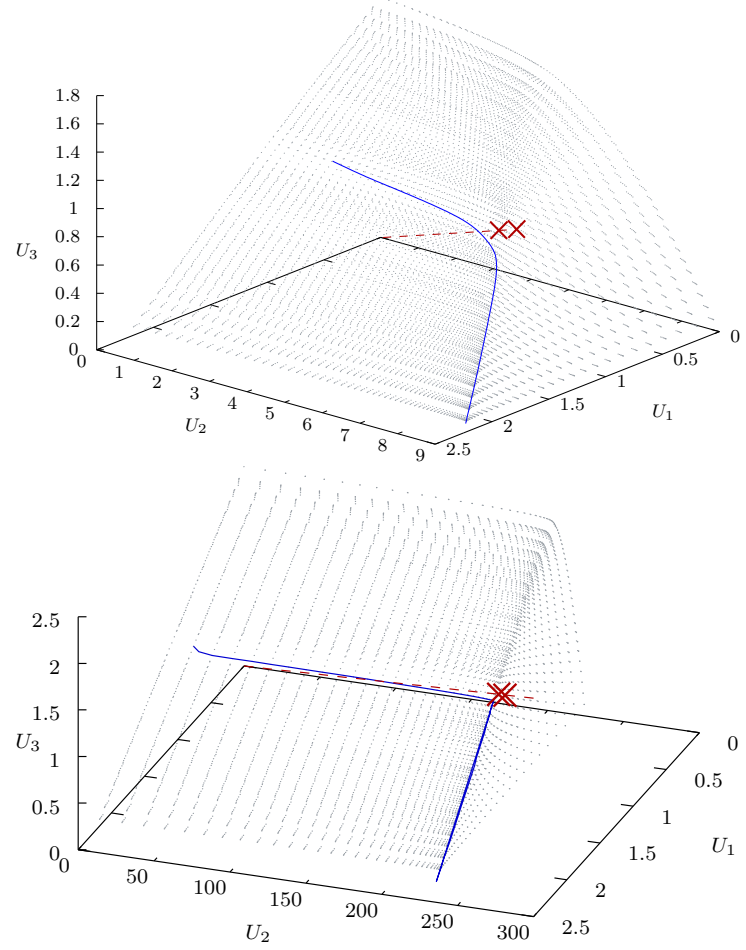

Fig. 4. The utility sets (for $\mathcal{N}=\{1,2,3\}$ ) in the flow control of a network of cases A (top) and B (bottom). For the cases A and B, respectively, the Nash proportionate fair Pareto optimal point and the Pareto optimal points superior to the Nash equilibrium point are not included in the set of the generalized fairness points with parameter $\alpha$ (that is, $\alpha$-fairness points)

fair Pareto optimal point is not included in the set of $\alpha$ fairness points, and that, in the network of case B (bottom), the Pareto optimal points superior to the Nash equilibrium point are not included in the set of the generalized fairness points with parameter $\alpha$ (that is, $\alpha$-fairness points) [7].

As anticipated from the fact that the $\alpha$-fairness depends only on one parameter $\alpha$, we can see that the set of $\alpha$-fairness points covers only a very small segment of the whole set of Pareto optimal points. From the examination of this figure, we see that there are cases where the generalized fairness with parameter $\alpha$ (that is, the $\alpha$-fairness) does not cover the Nash proportionate fairness nor the Nash equilibrium based fairness.

\section{Noncooperative Flow Control with Additive Costs}

In this subsection, we briefly touch on another case of each user's objective. Consider the network described in Section III-A, and assume that the cost per packet over link $k$ is given by the function $\left(1 / \mu_{i k}\right) T^{(k)}\left(\rho_{k}\right)$ (given by (2)) where

$$
\rho_{k}=\sum_{p} \rho_{p k}, \quad \rho_{i k}=Q_{i k} \lambda_{i} .
$$

The total cost paid by player $i$ is thus

$$
J_{i}(\lambda)=\lambda_{i} T_{i}=\sum_{l} \rho_{i l} T^{(l)}\left(\rho_{l}\right) .
$$

The utility for player $i$ is then given by

$$
U_{i}(\lambda)=R_{i}\left(\lambda_{i}\right)-a_{i} J_{i}(\lambda),
$$

where $R_{i}$ is concave in its argument and $a_{i}$ is a positive constant. Utilities with the above structure are common in telecommunication networks (see, for example, Alpcan and T. Başar [23], [24] that study special cases of such utilities). The existence of a Nash equilibrium, $\widetilde{\lambda} \in C$, has been shown [10]. It is seen that, if more than one user has the positive $\widetilde{\lambda}_{i}$ in a Nash equilibrium, it is strongly Pareto inefficient [10].

Corollary 2: For any inefficient Nash equilibrium flow control, there exists a Pareto-optimal flow control that is Pareto superior to the Nash equilibrium flow control.

[Proof] See [11].

\section{Concluding Remarks}

In this paper we have introduced a new fairness concept. First, we have presented a general framework in which a Pareto optimal allocation exists that is Pareto superior to any Pareto inefficient allocation. Then, we have considered a Pareto optimum allocation that is Pareto superior to an inefficient Nash equilibrium 'Nash-equilibrium-based fair.' We have also discussed the concept of the Nash-proportionately fair Pareto optimum. We have shown a framework for which the Nash-equilibrium-based fair allocation that achieves Nash-proportionate fairness most closely exists.

In particular, we have considered noncooperative flow control. We have firstly considered the power criterion for the utility of each user, and have shown a Nash-equilibriumbased fair Pareto optimal allocation for an inefficient Nash equilibrium. We have also shown the existence of a Nashproportionately fair Pareto optimum for the inefficient Nash equilibrium in the situation. We have then considered another utility of additive costs and have shown that a Nashequilibrium-based fair Pareto optimum exists for an inefficient Nash equilibrium.

\section{AcKNOWLEDGMENT}

The authors thank Professor Dinh The Luc for useful comments about the closedness of Pareto optimal sets.

\section{REFERENCES}

[1] J. F. Nash, Jr., “The bargaining problem,” Econometrica, vol. 18, pp. $155-162,1950$

[2] D. Bertsekas and R. Gallager, Data Networks, 2nd Ed., Prentice-Hall, Englewood Cliffs, 1992.

[3] F. P. Kelly, A. K. Maulloo, and D. K. H. Tan, "Rate control in communication networks: shadow prices, proportional fairness and stability," Journal of the Operational Research Society, vol. 49, pp. 237-252, 1998 .

[4] F. P. Kelly, "Charging and rate control for elastic traffic," European Transactions on Telecommunications, vol. 8, pp. 33-37, 1997.

[5] R. Jain, D. Chiu, and W. Hawe, "A quantitative measure of fairness and discrimination for resource allocation in shared computer systems," DEC Research Report TR-301, September 1984.

[6] R. Mazumdar, L. G. Mason, and C. Doulgligeris, "Fairness in network optimal flow control: Optimality of product forms," IEEE Trans. Communications, vol. 39, no. 5, pp. 775-782, May 1991.

[7] J. Mo and J. Walrand, "Fair end-to-end window-based congestion control," IEEE/ACM Trans. Networking, vol. 8, no. 5, pp. 556-567, Oct. 2000.

[8] T. Bonald, M. Jonckheere, and A. Proutière, "Insensitivie load balancing," in Proc. of ACM Sigmetrics/Performance, 2004.

[9] A. Giessler, J. Haanle, A. Konig, and E. Pade, "Free buffer allocation - An investigation by simulation," Comput. Networks, vol. 1, pp. 191204, 1978. 
[10] H. Kameda and E. Altman, "Inefficient noncooperation in networking games of common-pool resources," IEEE J. on Selected Areas in Communications, Special Issue on Game Theory in Communication Systems, vol. 29, pp. 1260-1268, Sept. 2008.

[11] H. Kameda, E. Altman, C. Touati, and A. Legrand, "Nash equilibrium based fairness," Tech. Rep., OSDP, Department of Computer Science, University of Tsukuba, 2009.

[12] H. Kameda, "Bounds on benefits and harms of adding connections to noncooperative networks," in NETWORKING 2004 - Proc. 3rd International IFIP-TC6 Networking Conference, Athens, Greece, May 9-14, 2004, Series: Lecture Notes in Computer Science, Vol. 3042 (N. Mitrou, et al., Eds.), Berlin, Germany, 2004, pp. 405-417, SpringerVerlag.

[13] A. Inoie, H. Kameda, and C. Touati, "Pareto set, fairness, and Nash equilibrium: A case study on load balancing," in Proc. 11th Int. Symp. Dynamic Games and Applications, Tucson, Arizona, Tucson, Arizona, Dec. 2004, pp. 386-393.

[14] O. Simeone and Y. Bar-Ness, "A game-theoretic view on the interference channel with random access," in Proc. 2nd IEEE International Symposium on New Frontiers in Dynamic Spectrum Access Networks, Dublin, April 2007, pp. 13-21, IEEE.

[15] T. Bonald and L. Massoulié, "Impact of fairness on Internet performance," in Proc. ACM Sigmetrics Conf., 2001, pp. 82-91.

[16] L. Massoulié and J. Roberts, "Bandwidth sharing: objectives and algorithms," in IEEE INFOCOM, 1999, pp. 1395-1403.

[17] H. Yaïche, R. R. Mazumdar, and C. Rosenberg, "A game theoretic framework for bandwidth allocation and pricing in broadband networks," IEEE/ACM Trans. Networking, vol. 8, no. 5, pp. 667-677, 2000.

[18] C. Touati, E. Altman, and J. Galtier, "Utility based fair bandwidth allocation," in Proc. NPDPA, Tsukuba, Japan, October 2002, pp. 126131, IASTED.

[19] E. Altman and H. Kameda, "Equilibria for multiclass routing in multi-agent networks," in Advances in Dynamic Games: Annals of International Society of Dynamic Games Vol. 7, A.S. Nowak and K. Szajowski, Eds., Boston, 2005, pp. 343-367, Birkhäuser, (An extended version of the paper that appeared in Proc. 40th IEEE Conference on Decision and Control (IEEE CDC'01), Orlando, U.S.A., pp. 604-609, Dec. 2001).

[20] H. Kameda, E. Altman, T. Kozawa, and Y. Hosokawa, "Braess-like paradoxes in distributed computer systems," IEEE Trans. Automatic Control, vol. 45, no. 9, pp. 1687-1691, 2000.

[21] H. Kameda and O. Pourtallier, "Paradoxes in distributed decisions on optimal load balancing for networks of homogeneous computers," $J$. ACM, vol. 49, no. 3, pp. 407-433, 2002.

[22] F. Baskett, K. Mani Chandy, R. Muntz, and F. G. Palacios, "Open, closed, and mixed networks of queues with different classes of customers," J. ACM, vol. 22, no. 2, pp. 248-260, 1975.

[23] T. Alpcan and T. Başar, "A game-theoretic framework for congestion control in general topology networks," in Proc. 4lth IEEE CDC, Las Vegas, Nevada, Dec. 10-13 2002, (Available at http://decision.csl.uiuc.edu/alpcan/publications.htm).

[24] T. Alpcan and T. Başar, "A utility-based congestion control scheme for Internet-style networks with delay," in Proc. IEEE Infocom, 2003, (Available at http://decision.csl.uiuc.edu/^alpcan/publications.htm). 